\title{
Sources d'influence de l'autoefficacité relative à un enseignement intégrant les TIC chez des enseignants du primaire
}

\section{Factors influencing teacher self-efficacy in relation to incorporating ICT at the elementary level \\ Fuentes de influencia de la auto-eficacia relativa a la enseñanza que integra las TIC entre los maestros de primaria}

\author{
Joanie Melançon, Sonia Lefebvre et Stéphane Thibodeau
}

Volume 41, numéro 1, printemps 2013

TIC et éducation : avantages, défis et perspectives futures

URI : https://id.erudit.org/iderudit/1015060ar

DOI : https://doi.org/10.7202/1015060ar

Aller au sommaire du numéro

\section{Éditeur(s)}

Association canadienne d'éducation de langue française

\section{ISSN}

0849-1089 (imprimé)

1916-8659 (numérique)

Découvrir la revue

Citer cet article

Melançon, J., Lefebvre, S. \& Thibodeau, S. (2013). Sources d'influence de l'autoefficacité relative à un enseignement intégrant les TIC chez des enseignants du primaire. Éducation et francophonie, 41(1), 70-93.

https://doi.org/10.7202/1015060ar

\section{Résumé de l'article}

Dans un contexte éducatif où l'intégration des technologies est prisée, on peut s'interroger sur la compétence des enseignants à intégrer les technologies de l'information et de la communication (TIC) (Mueller et al., 2008; Chai et al., 2011). Parmi les facteurs d'ordre personnel pouvant influencer le développement de cette compétence se trouve l'autoefficacité (Klassen et al., 2011; Wheatley, 2005). Cependant, les études qui s'intéressent aux facteurs d'influence de cette autoefficacité, dont celles de Bursal et Yigit (2012), de Kreijns et al. (2013) ainsi que de Robertson et Al-Zahrani (2012), affichent des résultats différents. Cet article présente donc une étude sur l'autoefficacité relative à un enseignement intégrant les TIC chez des enseignants du primaire. Plus précisément, il traite du niveau d'autoefficacité de ces enseignants et des sources d'influence de l'autoefficacité. Les résultats relatifs au niveau d'autoefficacité indiquent que la majorité des neuf enseignantes interrogées sont plutôt en accord avec le fait qu'elles sont en mesure d'intégrer les TIC dans leur enseignement et avec l'idée qu'un enseignement qui intègre les TIC apporte des retombées positives. Pour ce qui est des sources, ce sont l'expérience active de maîtrise ainsi que les états physiques et émotionnels qui semblent avoir une incidence plus marquée sur l'autoefficacité des participantes, alors que l'expérience vicariante et la persuasion verbale semblent y contribuer dans une moindre mesure. Ces résultats sont discutés à la lumière de la théorie sociocognitive de l'autoefficacité et d'études antérieures.
Tous droits réservés (C) Association canadienne d'éducation de langue française, 2013 cest protégé par la loi sur le droit d'auteur. L'utilisation des services d'Érudit (y compris la reproduction) est assujettie à sa politique d'utilisation que vous pouvez consulter en ligne. 


\section{Sources d'influence de I'autoefficacité relative à un enseignement intégrant les TIC chez des enseignants du primaire}

\section{Joanie MELANÇON}

Université du Québec à Trois-Rivières, Trois-Rivières, Canada

\section{SOnia LEFEBVRE}

Université du Québec à Trois-Rivières, Trois-Rivières, Canada

\section{Stéphane THIBODEAU}

Université du Québec à Trois-Rivières, Trois-Rivières, Canada

\section{RÉSUMÉ}

Dans un contexte éducatif où l'intégration des technologies est prisée, on peut s'interroger sur la compétence des enseignants à intégrer les technologies de l'information et de la communication (TIC) (Mueller et al., 2008; Chai et al., 2011). Parmi les facteurs d'ordre personnel pouvant influencer le développement de cette compétence se trouve l'autoefficacité (Klassen et al., 2011; Wheatley, 2005). Cependant, les études qui s'intéressent aux facteurs d'influence de cette autoefficacité, dont celles de Bursal et Yigit (2012), de Kreijns et al. (2013) ainsi que de Robertson et Al-Zahrani (2012), affichent des résultats différents. Cet article présente donc une étude sur l'autoefficacité relative à un enseignement intégrant les TIC chez des enseignants du 
primaire. Plus précisément, il traite du niveau d'autoefficacité de ces enseignants et des sources d'influence de l'autoefficacité. Les résultats relatifs au niveau d'autoefficacité indiquent que la majorité des neuf enseignantes interrogées sont plutôt en accord avec le fait qu'elles sont en mesure d'intégrer les TIC dans leur enseignement et avec l'idée qu'un enseignement qui intègre les TIC apporte des retombées positives. Pour ce qui est des sources, ce sont l'expérience active de maîtrise ainsi que les états physiques et émotionnels qui semblent avoir une incidence plus marquée sur l'autoefficacité des participantes, alors que l'expérience vicariante et la persuasion verbale semblent y contribuer dans une moindre mesure. Ces résultats sont discutés à la lumière de la théorie sociocognitive de l'autoefficacité et d'études antérieures.

\section{ABSTRACT}

\section{Factors influencing teacher self-efficacy in relation to incorporating ICT at the elementary level}

Joanie MELANÇON, M.A.

University of Québec in Trois-Rivières, Québec, Canada

Sonia LEFEBVRE, Ph. D.

University of Québec in Trois-Rivières, Québec, Canada

Stéphane THIBODEAU, Ph. D.

University of Québec in Trois-Rivières, Québec, Canada

In an educational context where incorporating technology is popular, the ability of teachers to integrate information and communication technology (ICT) remains questionable (Mueller et al., 2008; Chai et al., 2011). Self-efficacy is one of the personal factors that can influence the development of this competency (Klassen et al., 2011; Wheatley, 2005). However, studies on factors influencing self-efficacy, such as those of Bursal and Yigit (2012), Kreijns et al. (2013) and Robertson and Al-Zahrani (2012), show different results. This article presents a study on self-efficacy in relation to how elementary school teachers incorporate ICT. More specifically, it examines their level of self-efficacy and sources that influence it. The results related to self-efficacy levels show that the majority of the nine teachers interviewed agreed that they can use ICT in their teaching, and that incorporating ICT brings positive results. In terms of sources of self-efficacy, active mastery experiences and physical and emotional states seem to have a greater incidence on the participants' self-efficacy, while vicarious experience and verbal persuasion seem to make less of a contribution. These results are discussed in light of the socio-cognitive theory of self-efficacy and previous studies. 


\section{RESUMEN}

\section{Fuentes de influencia de la auto-eficacia relativa a la enseñanza que integra las TIC entre los maestros de primaria}

Joanie MELANÇON, M.A.

Universidad de Quebec en Trois-Rivières, Quebec, Canadá

Sonia LEFEBVRE, Ph. D.

Universidad de Quebec en Trois-Rivières, Quebec, Canadá

Stéphane THIBODEAU, Ph. D.

Universidad de Quebec en Trois-Rivières, Quebec, Canadá

En un contexto educativo en donde se valoriza la integración de las tecnologías, la competencia de los maestros para integrar las tecnologías de la información y de la comunicación (TIC) es cuestionable (Mueller et al., 2008; Chai et al., 2011). Entre los factores de orden personal que pueden influir el desarrollo de esa competencia se encuentra la auto-eficacia (Klassen et al.,2011; Wheatley, 2005). No obstante, los estudios que se interesan a los factores que influyen la auto-eficacia, entre ellos Bursal y Yigit (2021), de Kreijns et al. (2013) y el de Robertson y Al-Zahrani (2012), muestran resultados diferentes. Este artículo presenta un estudio sobre la auto-eficacia relacionada con la enseñanza que integra las TIC entre los maestros de primaria. Más precisamente, aborda el nivel de auto-eficacia y los factores que la influyen. Los resultados sobre el nivel de auto-eficacia muestran que la mayor parte de los nueve maestros interrogados están de acuerdo con el hecho de que pueden integrar las TIC en su enseñanza así como con la idea de que una enseñanza que integra las TIC acarrea repercusiones positivas. En lo concerniente a los factores, es la experiencia activa de control, así como los estados físicos y emotivos que parecen tener una incidencia más marcada sobre la auto-eficacia de los participantes, mientras que la experiencia vicariante y a la persuasión verbal parecen tener menor incidencia. Se discuten los resultados a la luz de la teoría socio-cognitiva de la auto-eficacia y de estudios anteriores.

\section{Introduction}

La mise en place du référentiel des compétences professionnelles des enseignants par le ministère de l'Éducation du Québec (2001) demande, entre autres, à l'enseignant d'intégrer les TIC dans son enseignement. Au même titre qu'il doit planifier ou piloter des activités d'apprentissage, l'enseignant compétent doit être en mesure d'intégrer les TIC en classe. C'est dans un contexte éducatif où l'importance des TIC est considérée que la présente étude s'intéresse à la compétence professionnelle en 
lien avec l'intégration des TIC. Dans le texte qui suit, il est question de la compétence professionnelle relative aux TIC, puis du concept d'autoefficacité. Les choix méthodologiques sont ensuite exposés. Enfin, les résultats sont présentés et discutés à la lumière de la théorie et d'écrits antérieurs.

\section{Compétence professionnelle relative aux TIC}

Bien que les TIC soient de plus en plus accessibles et variées, peu d'enseignants tendent à les intégrer efficacement pour bonifier leur enseignement ou pour soutenir l'apprentissage des élèves (Guzman et Nussbaum, 2009; Liu, 2011). En effet, la recherche montre que les TIC sont sous-utilisées en éducation, à l'échelle planétaire (Mueller, Wood, Willoughby, Ross et Specht, 2008). Selon Chai, Koh, Tsai et Tan (2011), les utilisations faites des TIC par les enseignants seraient critiques; les utilisations étant peu fréquentes et souvent destinées à transmettre de l'information plutôt que pour aider à la construction des connaissances des élèves. Comment expliquer une telle situation? Pour Hermans, Tondeur, van Braak et Valcke (2008), la réponse se trouve du côté des conceptions des enseignants pour l'enseignement et l'apprentissage. Il s'agirait d'un indicateur assez fiable des utilisations qui seront faites des outils technologiques. Pour d'autres, la question se pose plutôt sous l'angle du niveau de compétence des enseignants (Tsai et Chai, 2012; Villeneuve, Karsenti, Raby et Meunier, 2012).

L'enquête québécoise de Larose, Grenon et Palm (2004), menée auprès de 1180 enseignants en exercice, révèle que plusieurs enseignants ne possèdent que les compétences minimales nécessaires pour utiliser les logiciels de bureautique et les outils de communication. De surcroît, ce ne sont que $25 \%$ d'entre eux qui estiment employer les TIC régulièrement dans le but de soutenir l'apprentissage des élèves (Larose et al., 2004). Plus récemment, des données indiquent que seulement la moitié du personnel enseignant, des ordres primaire et secondaire, se considère comme étant suffisamment compétent pour intégrer les TIC avec les élèves (Ministère de l'Éducation, du Loisir et du Sport, 2010). Ces résultats rejoignent ceux obtenus par Goktas, Yildirim et Yildirim (2009) auprès de 1429 enseignants turcs du primaire et du secondaire. Même si ces derniers se perçoivent compétents pour manipuler les appareils informatisés (71,5\%), identifier les volets légaux et éthiques liés à un usage des TIC $(64,5 \%)$ et exploiter le traitement de texte dans un cadre personnel ou professionnel (68\%), ils ne sont que 33,1\% à se considérer comme aptes à exploiter les TIC pour soutenir l'enseignement (Goktas et al., 2009).

Chez les étudiants en formation initiale, la situation est comparable. À la lumière des travaux de Bidjang, Gauthier, Mellouki et Desbiens (2005), à peine 42,7\% d'entre eux ont atteint un niveau de compétence qualifié de très satisfaisant. Si les futurs enseignants possèdent une grande maîtrise des outils informatiques de base et que la majorité d'entre eux utilisent les TIC de façon régulière pour les activités de planification et de préparation de l'enseignement, ils ne représentent que $8,9 \%$ à utiliser fréquemment les outils informatisés avec les élèves (Karsenti, Raby, 
Villeneuve et Gauthier, 2007). Ainsi, la compétence professionnelle à intégrer les TIC à des fins pédagogiques est considérée, chez les futurs enseignants québécois du moins, comme partiellement maîtrisée (Villeneuve et al., 2012).

Par ailleurs, Hsu (2010) souligne qu'il n'existe pas de réel consensus sur ce qui devrait être pris en compte pour évaluer les enseignants dans l'intégration qu'ils font des TIC. Toutefois, parmi les facteurs qui semblent influencer le processus d'intégration des TIC se trouvent, entre autres, des facteurs liés au design pédagogique (Tsai et Chai, 2012), de même que des facteurs d'ordre personnel (Mueller et al., 2008; Raby, 2004). Or, la recherche en éducation s'intéresse depuis plusieurs années aux caractéristiques personnelles de l'enseignant, notamment à son autoefficacité (Klassen, Tze, Betts et Gordon, 2011; Wheatley, 2005).

\section{Facteurs d'influence de l'autoefficacité ${ }^{1}$ relative à l'intégration des TIC}

Une recension d'écrits montre que chez les étudiants en enseignement l'autoefficacité en lien avec un enseignement qui intègre les TIC est influencée par un certain nombre de facteurs: le niveau de maîtrise initial de la compétence TIC (Bursal et Yigit, 2012; Grenon, 2007; Robertson et Al-Zahrani, 2012), l'attitude à l'égard de l'intégration pédagogique des TIC (Sang, Valcke, van Braak et Tondeur, 2010), le fait d'être un étudiant finissant plutôt que débutant (Pamuk et Peker, 2009), le nombre d'heures passé à expérimenter Internet (Liang et Tsai, 2008), le fait d'avoir effectué des prises en charge lors de stages (Grenon, 2007; Van Dinther, Dochy et Segers, 2011) et le genre de l'enseignant associé au cours de ces mêmes stages (Grenon, 2007). Également, d'autres facteurs d'influence sont rapportés dans les travaux tels que le fait d'être issu d'un milieu socioéconomique élevé (Bursal et Yigit, 2012), la présence d'un cours TIC lors de la formation initiale (Robertson et Al-Zahrani, 2012; Van Dinther et al., 2011) et les sources d'autoefficacité (Van Dinther et al., 2011).

Du côté des enseignants en exercice, il existe aussi des facteurs d'influence de leur autoefficacité envers un enseignement qui intègre les TIC. Il s'agit, entre autres, de l'autoefficacité générale de l'enseignant (Paraskeva, Bouta et Papagianni, 2008), de sa facilité à utiliser les TIC (Shiue, 2007), du contrôle perçu envers les TIC (Shiue, 2007) et de son intention de les intégrer dans son enseignement (Shiue, 2007; Van Acker, Van Buuren, Kreijns et Vermeulen, 2011). D'autres facteurs d'influence ressortent dans les écrits, notamment le fait de détenir des connaissances et des aptitudes liées aux TIC (Kreijns, Van Acker, Vermeulen et Van Buuren, 2013; Van Acker et al., 2011), l'attitude à l'égard de ces outils, la perception d'avantages à les intégrer dans sa pratique pédagogique et les états physiques, dont le sentiment d'anxiété (Van Acker et al., 2011).

Même si plusieurs des études recensées s'intéressent à l'autoefficacité comme facteur d'influence de l'intégration des TIC dans un contexte scolaire (voir Bursal et Yigit, 2012; Grenon, 2007; Kreijns et al., 2013; Liang et Tsai, 2008; Pamuk et Peker,

1. Dans son ouvrage de référence de 2007, Bandura utilise plusieurs synonymes pour exprimer la même réalité: autoefficacité, efficacité personnelle, croyances d'efficacité, attentes d'efficacité, etc. Dans cet article, c'est le terme autoefficacité qui est utilisé. 
2009; Paraskeva et al., 2008; Robertson et Al-Zahrani, 2012; Sang et al., 2010; Shiue, 2007; Van Acker et al., 2011; Van Dinther et al., 2011), peu ont étudié de façon systématique les sources d'autoefficacité (Gloudemans, Schalk, Reynaert et Braeken, 2013). Dans les résultats des travaux qui se sont penchés sur le sujet, l'importance accordée à chacune des sources d'autoefficacité varie d'une étude à l'autre. En effet, certaines recherches rapportent l'importance de l'expérience active de maîtrise (Al-Awidi et Alghazo, 2012; Britner et Pajares, 2006; Kiran et Sungur, 2012; Usher et Pajares, 2009; Van Dinther et al., 2011; Zeldin, Britner et Pajares, 2008), de l'expérience vicariante (Al-Awidi et Alghazo, 2012; Hodges et Murphy, 2009; Van Dinther et al., 2011; Wang, Ertmer et Newby, 2004; Zeldin et Pajares, 2000), de la persuasion verbale (Van Dinther et al., 2011; Zeldin et Pajares, 2000), de même que des états physiques et émotionnels (Hodges et Murphy, 2009; Van Acker et al., 2011).

À la lumière de ces résultats, il est possible de constater l'absence de consensus concernant l'importance de chacune des sources d'autoefficacité. De plus, certains éléments varient d'une étude à l'autre. Par exemple, les études concernent tantôt les étudiants en enseignement (voir Bursal et Yigit, 2012; Grenon, 2007; Liang et Tsai, 2008; Pamuk et Peker, 2009; Robertson et Al-Zahrani, 2012, Sang et al., 2010; Van Dinther et al., 2011), tantôt les enseignants en exercice (voir Kreijns et al., 2013; Paraskeva et al., 2008; Shiue, 2007; Van Acker et al., 2011). De plus, si ces études définissent l'autoefficacité à partir de la théorie sociocognitive de Bandura (2007), elles n'utilisent pas toutes des instruments qui sont totalement cohérents avec cette théorie pour mesurer son niveau (Usher et Pajares, 2009). Enfin, certaines ne s'appuient pas sur ce cadre pour analyser les facteurs d'influence de l'autoefficacité. Par conséquent, il apparaît pertinent de s'intéresser à ces facteurs à la lumière des travaux de Bandura, notamment dans un contexte d'enseignement qui intègre les TIC (Shiue, 2007). Dans cette perspective, la recherche s'intéresse à l'autoefficacité relative à un enseignement qui intègre les TIC chez des enseignants. Précisément, elle poursuit les deux objectifs suivants: 1) déterminer leur niveau d'autoefficacité relative à un enseignement intégrant les TIC et 2) identifier les sources d'influence de leur autoefficacité.

\section{Concept d'autoefficacité}

Les assises théoriques de la recherche reposent essentiellement sur la théorie sociocognitive de Bandura, car celle-ci est couramment utilisée dans les travaux portant sur l'autoefficacité des enseignants. L'autoefficacité détermine le comportement humain et fait référence aux croyances d'un individu quant à ses aptitudes à organiser et à exécuter les actions requises pour produire les performances escomptées (Bandura, 1997 et 2007).

Les attentes d'efficacité (autoefficacité) et les attentes de résultats, qui animent l'individu, sont deux concepts distincts. Alors que l'autoefficacité renvoie aux croyances de l'individu relativement à sa capacité à réaliser une performance, les attentes de résultats sont en lien avec les croyances que la performance va produire des effets positifs ou négatifs (Bandura, 1997 et 2007). 
Bien que certains proposent un modèle à cinq dimensions pour expliquer le développement de l'autoefficacité, les preuves empiriques à cet effet sont encore insuffisantes (Gloudemans et al., 2013). L'idée de Bandura voulant que l'autoefficacité se modifie sous l'influence de quatre sources d'information demeure donc la plus largement acceptée (Gloudemans et al., 2013; Van Dinther et al., 2011). Ces sources sont l'expérience active de maîtrise, l'expérience vicariante, la persuasion verbale et les états physiques et émotionnels. L'individu doit nécessairement peser et traiter les informations provenant de ces sources afin de les intégrer (Anderson et Betz, 2001; Bandura, 1997, 2007; Bong et Skaalvik, 2003; Hodges et Murphy, 2009; Palmer, 2006; Usher et Pajares, 2006, 2009; Van Dinther et al., 2011).

\section{Expérience active de maîtrise}

L'expérience active de maîtrise correspond aux succès et aux échecs que vit la personne. Cette source s'avère être la plus influente, parce qu'elle illustre le plus clairement que l'individu est en mesure de rassembler, ou non, ce qui est nécessaire pour vivre des succès (Bandura, 1997 et 2007; Palmer, 2006). L'expérience active de maîtrise est influencée par cinq thèmes, dont l'aide reçue/donnée, l'auto-observation sélective, les circonstances, l'effort fourni et l'adéquation des ressources.

Lorsque l'individu reçoit de l'aide pour exécuter une tâche, il aura tendance à interpréter son succès en lien avec cette aide plutôt qu'avec ses aptitudes personnelles. Il se peut aussi que l'individu concentre son attention davantage vers les succès ou encore les échecs vécus; c'est ce que l'on appelle l'auto-observation sélective. De même, les circonstances peuvent inhiber ou faciliter la réalisation d'une activité. Un échec qui survient dans des circonstances peu propices à la réussite produit moins d'impact sur l'autoefficacité de l'individu que s'il était advenu dans des circonstances favorables. Également, les résultats sont interprétés par l'individu en tenant compte de l'effort fourni pour exécuter la tâche. Si la personne déploie un minimum d'efforts dans l'exécution d'une tâche et qu'elle connaît des succès, alors que la tâche est perçue difficile par les pairs, elle aura une propension à croire qu'elle possède de grandes aptitudes. Toutefois, si l'individu fournit des efforts laborieux en lien avec une tâche et qu'il vit des succès, il considérera qu'il a de moins grandes aptitudes que s'il avait employé peu d'efforts pour y parvenir. Enfin, les ressources financières, matérielles et personnelles représentent d'autres facteurs susceptibles d'inhiber ou encore de faciliter la réalisation d'une tâche (Bandura, 1997, 2007).

\section{Expérience vicariante}

L'expérience vicariante concerne l'apprentissage par l'observation de modèles. Grâce au modelage ainsi réalisé, l'individu développe des compétences et acquiert des connaissances. L'individu peut apprendre au contact d'un modèle soit par un enseignement verbal de la tâche, soit par l'observation du modèle dans l'action (Bandura, 1997 et 2007; Van Dinther et al., 2011). Trois thèmes influencent l'expérience vicariante: la compétence du modèle, la similitude de caractéristiques entre les individus et la similitude de performance. 
Bandura (2007) soutient que le niveau de compétence du modèle est le thème le plus important en ce qui concerne l'expérience vicariante. Quand l'individu a beaucoup à apprendre et que le modèle a beaucoup à enseigner, la compétence du modèle devient alors un facteur d'influence important. De même, si un individu possède une ou des caractéristiques personnelles similaires à celles de son modèle, ce dernier s'identifiera à lui. Ainsi, les succès ou encore les échecs vécus par le modèle représenteront un indice de ses propres succès ou échecs. En effet, si l'individu est en contact avec un modèle qui obtient une performance similaire à la sienne, les résultats obtenus par le modèle seront perçus comme un diagnostic de ses propres aptitudes. Si l'individu compare sa performance avec celle d'un modèle qu'il estime similaire à lui-même, cela peut augmenter sa croyance voulant qu'il puisse lui aussi en arriver à de tels succès ou échecs. Toutefois, si la comparaison de la performance se base sur les succès d'un modèle jugé plus compétent par l'individu, cela peut occasionner de la dévalorisation et du découragement chez ce dernier (Bandura, 1997, 2007).

\section{Persuasion verbale}

La persuasion verbale concerne les propos à l'endroit d'un individu dans le but de le convaincre, ou de le dissuader, qu'il possède les capacités à maîtriser une activité. Lorsqu'une personne tente d'en convaincre une autre, cette dernière a plus de chances de produire un effort supplémentaire et de le maintenir. Cependant, si une personne tente d'en dissuader une autre, cette dernière aura, par la suite, tendance à éviter les situations qui représentent un défi et à renoncer rapidement lorsqu'elle fera face à une épreuve (Bandura, 1997, 2007; Bong et Skaalvik, 2003; Van Dinther et al., 2011). Quatre thèmes influencent la persuasion verbale: les propos basés sur l'aptitude, la similitude avec les caractéristiques, la crédibilité de la personne qui tente de convaincre ou de dissuader et l'effort fourni.

Quand une personne tente d'en persuader une autre à propos de l'accomplissement d'une tâche, faire allusion aux aptitudes ou compétences de cette dernière, plutôt qu'aux efforts fournis, contribue à accroître son autoefficacité. L'individu accordera plus ou moins d'importance aux propos persuasifs en fonction de la similitude de caractéristiques personnelles qu'il possède avec la personne qui tente de le persuader. Si l'individu considère que cette dernière possède des caractéristiques similaires aux siennes, les propos seront perçus plus importants. La valeur accordée aux propos est liée, entre autres, à la crédibilité de la personne qui tente de persuader et lui confère un certain statut qui influencera favorablement le traitement des propos reçus par l'individu (Bandura, 1997, 2007).

\section{États physiques et émotionnels}

L'individu évalue ses propres capacités à la lumière des données somatiques transmises par ses états physiques et émotionnels. Il perçoit qu'il possède ou non la capacité à effectuer une tâche donnée, en fonction de ce qu'il ressent sur les plans physique et émotionnel. De cette façon, si l'individu se sent bien en effectuant, ou à l'idée de devoir effectuer, une tâche, il interprètera qu'il possède les capacités requises pour la réaliser. À l'inverse, s'il ne se sent pas bien, il interprètera qu'il ne possède pas 
les capacités requises pour réussir (Bandura, 1997, 2007; Van Dinther et al., 2011). Deux thèmes influencent les états physiques et émotionnels : les activateurs environnementaux et le sentiment de compétence.

Les activateurs environnementaux influencent l'interprétation que la personne fait des indicateurs physiques et émotionnels. Par exemple, l'accélération du rythme cardiaque peut être causée par un grand stress ressenti ou lié à de l'euphorie selon la situation. Ce ne sont pas tant les états en eux-mêmes qui sont importants que la façon dont ils sont perçus et interprétés. En outre, un traitement positif des états ressentis devrait faire augmenter le sentiment de compétence de l'individu, ainsi que son autoefficacité. À l'inverse, un traitement négatif des états ressentis devrait faire diminuer le sentiment de compétence de l'individu, ainsi que son autoefficacité (Bandura, 1997 et 2007).

\section{Intégration de l'information}

L'intégration de l'information ne constitue pas une des sources de l'autoefficacité, mais bien le traitement que l'individu fait des informations provenant des quatre sources mentionnées ci-dessus. Ces informations sont utiles quand la personne les pèse et les traite pour former son autoefficacité. Certains individus intègrent les diverses informations de manière additive. Dans ce cas, si toutes les informations sont positives, l'individu va considérer l'addition de celles-ci et en tirer une conclusion positive. $\mathrm{Si}$, au contraire, les informations sont négatives, l'individu va alors interpréter cette addition en lui accordant un sens négatif. Pour certains, la pondération attribuée aux différents facteurs diffère selon l'importance donnée par chacun des individus, alors que d'autres «utilisent une règle de combinaison multiplicative où l'impact conjoint des facteurs sur l'efficacité est plus grand que leur simple effet additif" (Bandura, 2007, p. 175). Finalement, d'autres individus vont effectuer une combinaison de manière configurée, ce qui veut dire qu'ils vont accorder un poids différent à un facteur spécifique selon les sources d'autoefficacité (Bandura, 1997 et 2007).

\section{Autoefficacité relative à un enseignement qui intègre les TIC}

Gibson et Dembo (1984) sont les pionniers en ce qui concerne l'autoefficacité étudiée sous l'angle de l'enseignement. Selon eux, les croyances d'efficacité générale ${ }^{2}$ font référence aux croyances de l'enseignant voulant que l'enseignement puisse apporter des retombées positives. Ce concept, comme le présente Bandura (2007), représente les attentes de résultats. Quant aux croyances d'efficacité personnelle, elles renvoient à une certaine forme d'autoévaluation, où l'enseignant perçoit sa propre capacité à influencer l'apprentissage des élèves. Pour Bandura (2007), cela correspond aux attentes d'efficacité.

Dans un contexte d'enseignement qui exploite les TIC, l'autoefficacité correspond aux aptitudes de l'enseignant à organiser et à exécuter les actions requises pour

2. Il est à noter que le terme "croyances d'efficacité» est utilisé dans le seul but de ne pas déformer la théorie telle que la proposent Gibson et Dembo (1984). 
produire une performance dans son enseignement qui intègre les TIC. L'autoefficacité relative à un enseignement qui intègre les TIC est en lien avec ce que l'enseignant se croit en mesure de faire dans une variété de situations qui nécessitent le recours aux TIC. Un enseignant possède des croyances d'efficacité personnelles élevées s'il se croit capable d'intégrer les TIC dans son enseignement (Mélançon, 2012).

\section{Choix méthodologiques}

Sont décrits ici le contexte de l'étude, les participants, les outils de collecte des données, de même que le traitement des données.

\section{Contexte de l'étude}

L'étude a été conduite dans le cadre d'une recherche plus vaste, menée dans une école primaire de la région de la Mauricie, le projet de journal virtuel Le Racont'art. S'inscrivant dans le projet éducatif de l'école, il a été mis sur pied dans le but d'accroître les pratiques d'intégration des TIC des enseignants de l'école. Les enseignants et leurs élèves étaient invités à rédiger des articles et à les publier sur le site du journal virtuel selon leur convenance et à leur propre rythme, minimalement une fois par mois.

\section{Participantes}

De l'ensemble de l'équipe-école, neuf enseignantes se sont montrées désireuses de participer à l'étude. Alors que cinq d'entre elles enseignent au $1^{\mathrm{er}}, 2^{\mathrm{e}}$ ou $3^{\mathrm{e}}$ cycle de l'enseignement ordinaire, quatre œuvrent auprès d'élèves ayant des besoins particuliers. Les enseignantes ont entre 5 et 25 ans d'expérience dans la profession.

\section{Outils de collecte des données}

Afin de déterminer le niveau d'autoefficacité relatif à un enseignement intégrant les TIC chez des enseignants, l'Échelle du sentiment d'efficacité des enseignants à l'égard de l'intégration des technologies de l'information et des communications en classe (SÉTIC), de Dussault, Deaudelin, Brodeur et Richer (2002), a été utilisée. L'outil comporte 23 énoncés, dont 13 portent sur les attentes d'efficacité, alors que 10 traitent des attentes de résultats. L'outil présente une bonne stabilité temporelle $\left(r_{\text {test-retest }} 3\right.$ semaines $\left.=0,72\right)$ ainsi qu'une bonne consistance interne $(\alpha=0,92)$. Pour chacun des énoncés, les enseignants indiquent leur niveau d'accord sur une échelle en quatre points: (1) Totalement en accord; (2) Plutôt en accord; (3) Plutôt en désaccord et (4) Totalement en désaccord. Les enseignantes ont répondu à ce questionnaire en une quinzaine de minutes.

Dans le but d'identifier les sources qui influencent l'autoefficacité relative à l'enseignement intégrant les TIC d'enseignants, les données ont été recueillies à l'aide d'une entrevue semi-dirigée d'une durée moyenne de 30 minutes. Le canevas compte neuf questions et amène l'enseignante à décrire l'incidence de chacune des quatre sources d'influence sur son autoefficacité relative à un enseignement qui 
intègre les TIC. Les questions ont été élaborées en cohérence avec la théorie de Bandura (2007).

\section{Traitement des données}

Le traitement des données issues du questionnaire SÉTIC a été réalisé à l'aide du logiciel SPSS. Ce dernier permet de calculer les moyennes des attentes d'efficacité et des attentes de résultats des participantes. Afin de faciliter le traitement des données et d'assurer une cohérence, les moyennes des attentes d'efficacité et des attentes de résultats ont été rapportées en fonction des quatre points de l'échelle du SÉTIC. Ainsi, les scores allant de 1 à 1,5 correspondent à «Totalement en désaccord», les scores se situant de 1,51 à 2,5 se retrouvent dans la catégorie "Plutôt en désaccord", les scores qui se situent entre 2,51 et 3,5 font partie de la catégorie «Plutôt en accord" et les scores allant de 3,51 à 4 appartiennent à la catégorie «Totalement en accord».

En ce qui a trait aux entrevues, les unités de sens ont été analysées à l'aide d'un système catégoriel élaboré à partir de la théorie de Bandura (2007). Comme le mentionne Van der Maren (1996), certains analystes miniaturisent les unités quand vient le temps de segmenter, alors que d'autres réduisent la liste à ce qui saute aux yeux. Dans ce cas-ci, la segmentation a été faite de manière à conserver une idée. Cependant, les unités de sens ont été traitées en ayant en tête l'ensemble des propos de l'entrevue. De façon plus précise, les unités de sens ont été catégorisées et traitées en lien avec les quatre sources d'autoefficacité qui sont, faut-il le rappeler, l'expérience active de maîtrise, l'expérience vicariante, la persuasion verbale et les états physiques et émotionnels. Chaque extrait segmenté était d'abord accolé à l'une des quatre sources d'autoefficacité. Ensuite, on l'associait de manière plus précise à l'un des thèmes de cette source. Par exemple, un extrait pouvait être lié à la source de l'expérience active de maîtrise, pour ensuite être associé à l'un des thèmes, soit la finalité. Au terme de l'exercice, un double codage a été effectué afin d'obtenir l'accord interjuges. Le logiciel libre Weft Qda a été utilisé afin de procéder à l'analyse qualitative. L'emploi de logiciels d'analyse qualitative, tels que Weft Qda, constitue un moyen d'objectiver les données (Hamel, 2010).

\section{Résultats}

Ce sont d'abord les résultats concernant le niveau d'autoefficacité qui sont présentés. Ensuite, il est question des résultats relatifs aux sources d'autoefficacité, plus précisément des thèmes abordés pour chacune de ces sources.

\section{Niveau d'autoefficacité}

Les résultats de la figure 1 montrent que la moyenne des attentes d'efficacité des participantes se situe à $2,73 / 4$, alors que la moyenne des attentes de résultats est de $2,76 / 4$. 
Figure 1. Niveau d'autoefficacité relatif à un enseignement intégrant les TIC d'enseignantes du primaire

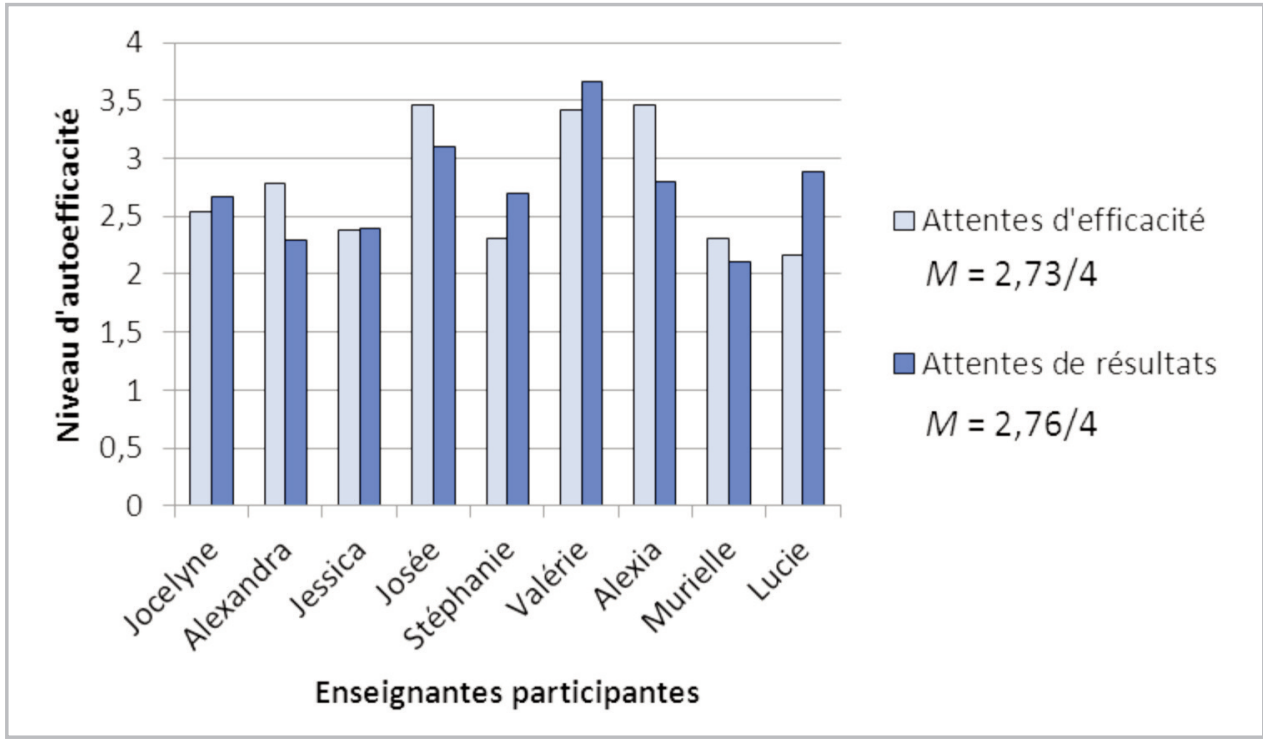

Au regard des attentes d'efficacité, cinq des enseignantes ${ }^{3}$ (Jocelyne, Alexandra, Josée, Valérie et Alexia) sont plutôt en accord avec l'idée d'être en mesure d'intégrer les TIC dans leur enseignement, alors que quatre d'entre elles (Jessica, Stéphanie, Murielle et Lucie) semblent être plutôt en désaccord. En ce qui concerne les attentes de résultats, une des enseignantes (Valérie) est totalement en accord avec l'idée qu'un enseignement qui intègre les TIC apporte des retombées positives et cinq (Jocelyne, Josée, Stéphanie, Alexia et Lucie) sont plutôt en accord avec cette même idée. Toutefois, trois des participantes (Alexandra, Jessica et Murielle) sont plutôt en désaccord avec le fait qu'un enseignement qui intègre les TIC apporte des retombées positives.

\section{Sources d'autoefficacité}

La figure 2 illustre la fréquence des propos des participantes pour chacune des sources qui influencent l'autoefficacité relative à l'enseignement intégrant les TIC.

3. Les prénoms des participantes sont fictifs. 
Figure 2. Fréquence des propos rapportés par les participantes selon leur appartenance aux sources qui influencent l'autoefficacité relative à un enseignement intégrant les TIC

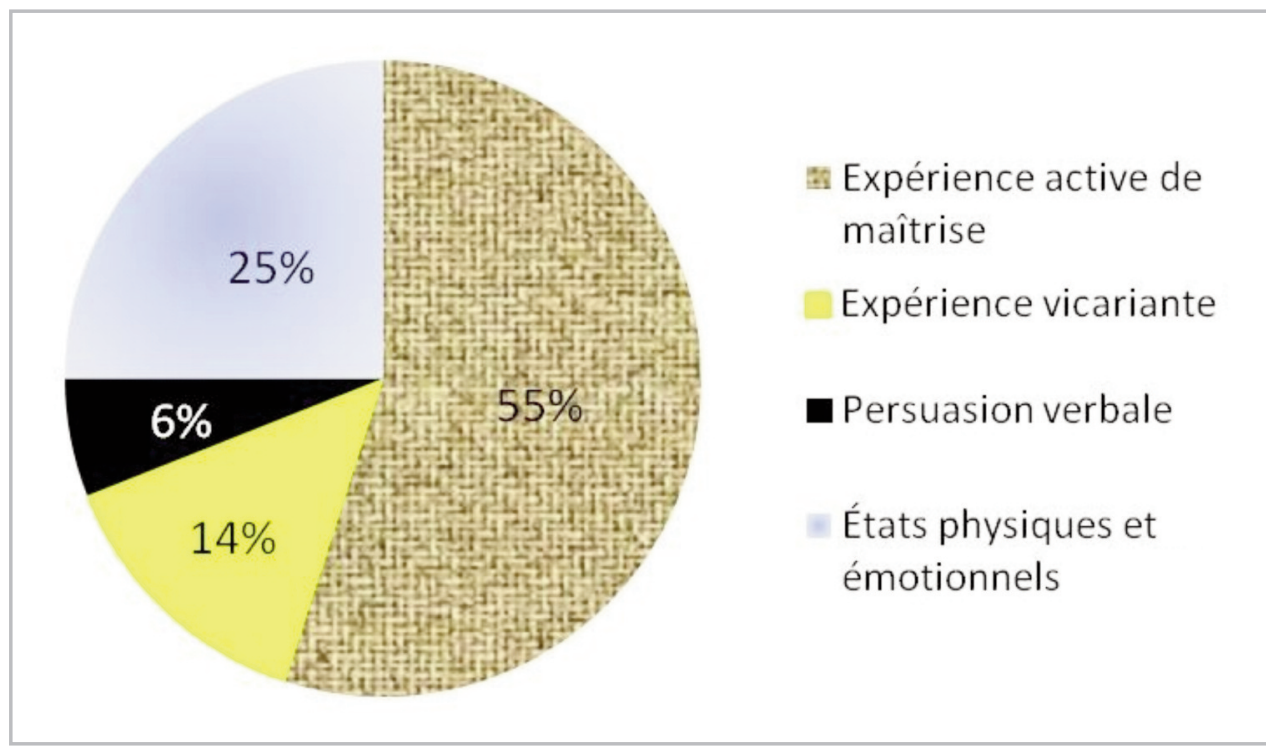

D'emblée, on remarque que la majorité des propos se rattachent à l'expérience active de maîtrise (55\%). Les succès et les échecs vécus par les participantes, lorsque celles-ci intègrent les TIC dans leur enseignement, sont évoqués plus souvent que toute autre source. Ensuite, ce sont les états physiques et émotionnels (25\%) qui sont le plus rapportés par les participantes. Les états ressentis (stress, palpitations, anxiété, transpiration abondante, etc.) en situation d'intégration des TIC constituent donc également une source importante de leur autoefficacité. Pour leur part, l'expérience vicariante $(14 \%)$ et la persuasion verbale $(6 \%)$ sont rapportées dans de moindres proportions. Ainsi, d'après les résultats obtenus, tant le modelage au contact d'un modèle qui intègre les TIC que les propos persuasifs reçus au regard de leur compétence à intégrer les TIC semblent être des sources moins privilégiées pour influencer leur autoefficacité liée à un enseignement qui intègre les TIC.

\section{Thèmes abordés pour chacune des sources de l'autoefficacité Expérience active de maîtrise}

Ainsi que le montre la figure 3, les participantes abordent l'expérience active de maîtrise en des termes liés à la finalité (32\%) poursuivie au sein d'une activité qui intègre les TIC. Par exemple, pour Josée, il importe de poursuivre un but pédagogique lorsqu'elle recourt aux TIC: «... je ne fais jamais les choses seulement pour occuper les élèves. C'est toujours dans un but» (Josée 70-72). Cibler des intentions pédagogiques, 
et les atteindre, contribue ainsi à renouveler ses expériences pédagogiques avec les TIC. Les enseignantes se préoccupent aussi des circonstances ou facteurs $(26,1 \%)$ influençant les expériences faites des TIC, comme le temps investi pour se former, s'approprier les outils et planifier des activités. Les ressources matérielles (18\%) disponibles à l'école représentent un autre facteur ayant une incidence sur les expériences d'intégration des TIC faites par les enseignantes.

Figure 3. Fréquence des thèmes abordés relativement à la source de l'expérience active de maîtrise pour l'ensemble des participantes

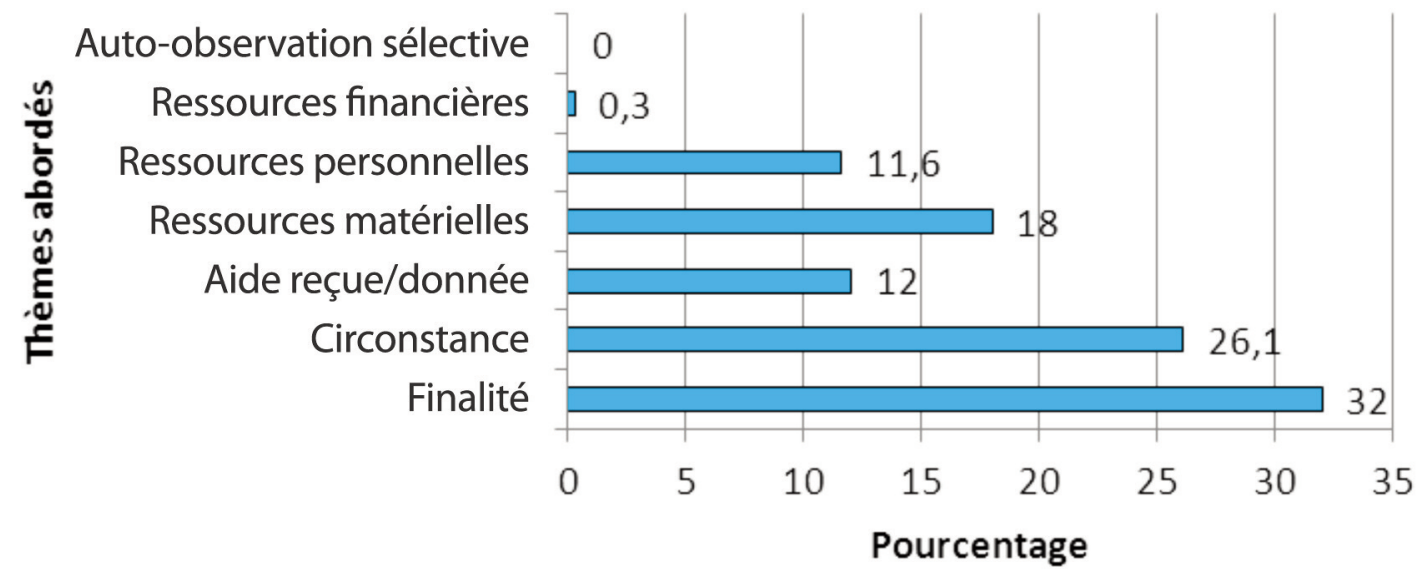

Dans des proportions moindres, les enseignantes abordent l'aide qu'elles peuvent recevoir ou donner (12\%) en cours de déroulement d'une activité qui exploite les TIC de même que leur bagage personnel et pédagogique $(11,6 \%)$ comme des facteurs d'influence. Par contre, l'auto-observation sélective $(0 \%)$ ne semble pas constituer un élément influençant l'exploitation qu'elles font des TIC dans un cadre pédagogique.

\section{Expérience vicariante}

Quand il s'agit de l'observation d'un modèle pour se former à l'exploitation des TIC à des fins pédagogiques, la figure 4 montre que la compétence de ce dernier $(79,4 \%)$ ressort comme l'élément le plus influent pour accroître l'autoefficacité à intégrer les TIC. L'exemple qui suit illustre la réaction d'une participante lorsqu'elle fait référence au conseiller pédagogique en TIC qui lui donne des formations: « $L a$ variété de choses qu'il peut nous montrer, c'est stimulant, c'est dynamique, c'est différent. Il y a toujours un petit quelque chose de plaisant. J'aime bien ça» (Alexia 112-113). 
Figure 4. Fréquence des thèmes abordés relativement à la source de l'expérience vicariante pour l'ensemble des participantes

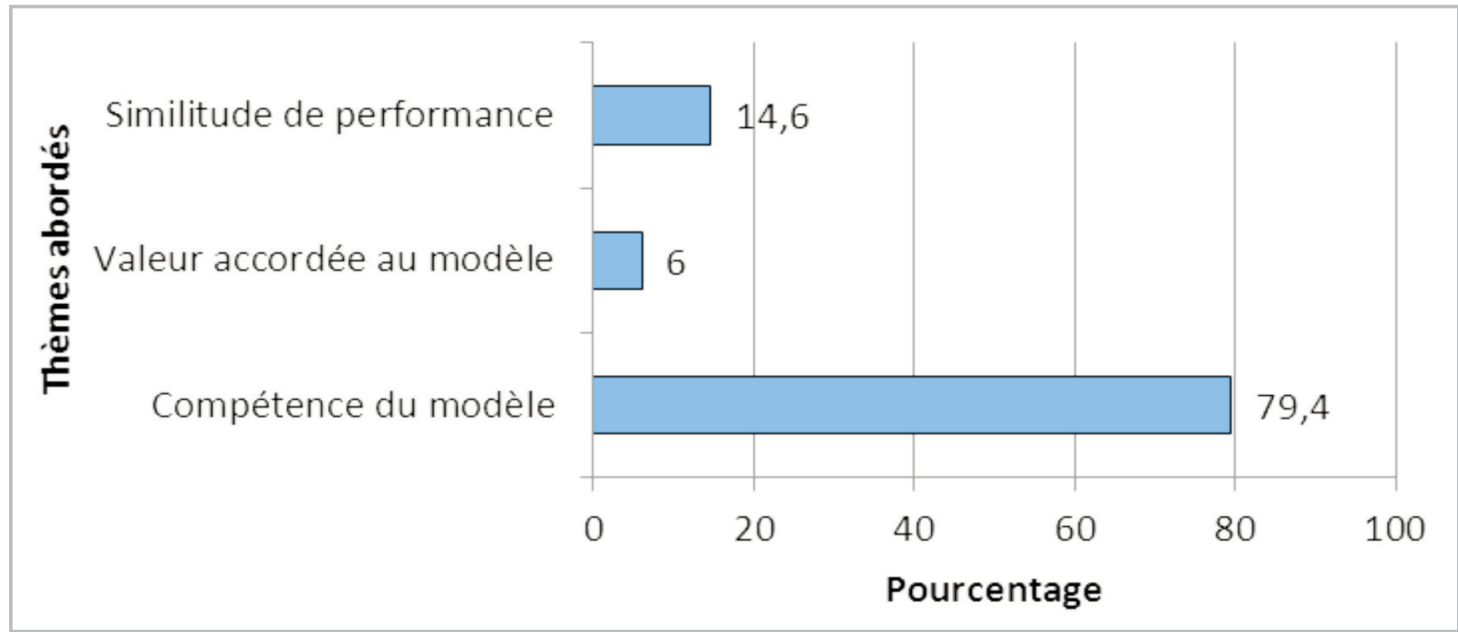

Dans une proportion plus faible (14,6\%), comparer ses réalisations à l'aide des TIC avec celles de son modèle représente un autre élément qui influe sur l'autoefficacité des enseignantes. Enfin, la valeur accordée au modèle (6\%) peut être un élément contribuant à accroître l'autoefficacité à intégrer les TIC dans la mesure où, comme c'est le cas avec Jocelyne, l'enseignante a de l'estime pour son modèle. "Valérie, c'est une personne que j'aime beaucoup. Elle donne du temps, puis elle s'investit là-dedans. Donc ça m’incite à participer» (Jocelyne 88-90).

\section{Persuasion verbale}

En ce qui a trait à la persuasion verbale (voir la figure 5), le traitement $(36,4 \%)$ que fait l'enseignant des propos reçus d'autrui, et qui visent à le convaincre ou à le dissuader de ses capacités à intégrer les TIC, joue un rôle dans le développement de l'autoefficacité à intégrer les TIC. L'extrait suivant mentionne qu'Alexandra souhaite continuer d'intégrer les TIC en raison des encouragements qu'elle reçoit: "Ça me donne le petit encouragement de l'essayer. Je vais essayer de continuer parce que je reçois des encouragements» (Alexandra 204-206). Dans le même sens, des propos qui témoignent de l'aptitude $(33,3 \%)$ de l'enseignant contribuent à accroître l'autoefficacité à intégrer les TIC. 
Figure 5. Fréquence des thèmes abordés relativement à la source de la persuasion verbale pour l'ensemble des participantes

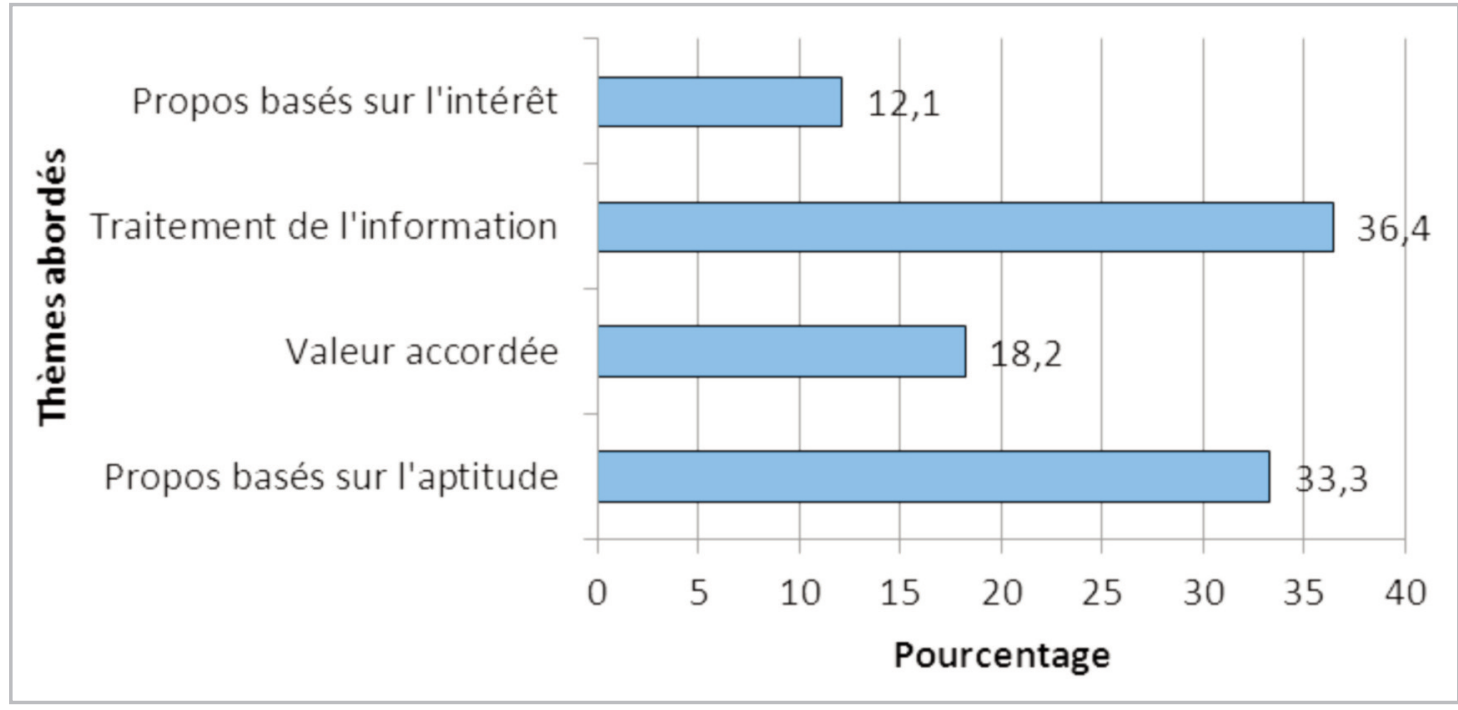

Quant à la valeur accordée aux propos du modèle $(18,2 \%)$ et aux propos basés sur l'intérêt (12,1\%), bien qu'ils soient importants pour favoriser l'autoefficacité, peu d'unités de sens ont été formulées au regard de chacun de ces thèmes.

\section{États physiques et émotionnels}

La figure 6 montre bien l'influence des éléments de l'environnement $(61,1 \%)$ dans l'interprétation des manifestations ressenties par l'enseignant lors du déroulement d'activités pédagogiques qui intègrent les TIC. Lorsque Murielle mentionne que le fait d'intégrer les TIC en présence d'un groupe d'élèves active son état de manière négative, cela représente un exemple qui traduit bien son état émotionnel: "Sur les nerfs. Si j'ai un groupe avec moi au laboratoire, je suis dans les nerfs. Je ne suis pas bien. J'ai hâte que ça finisse.» (Murielle 185-186). Dans ce dernier cas, l'interprétation des états physiques et émotionnels ne favorise pas, chez l'enseignante, le développement de son autoefficacité. 
Figure 6. Fréquence des thèmes abordés relativement à la source des états physiques et émotionnels pour l'ensemble des participantes

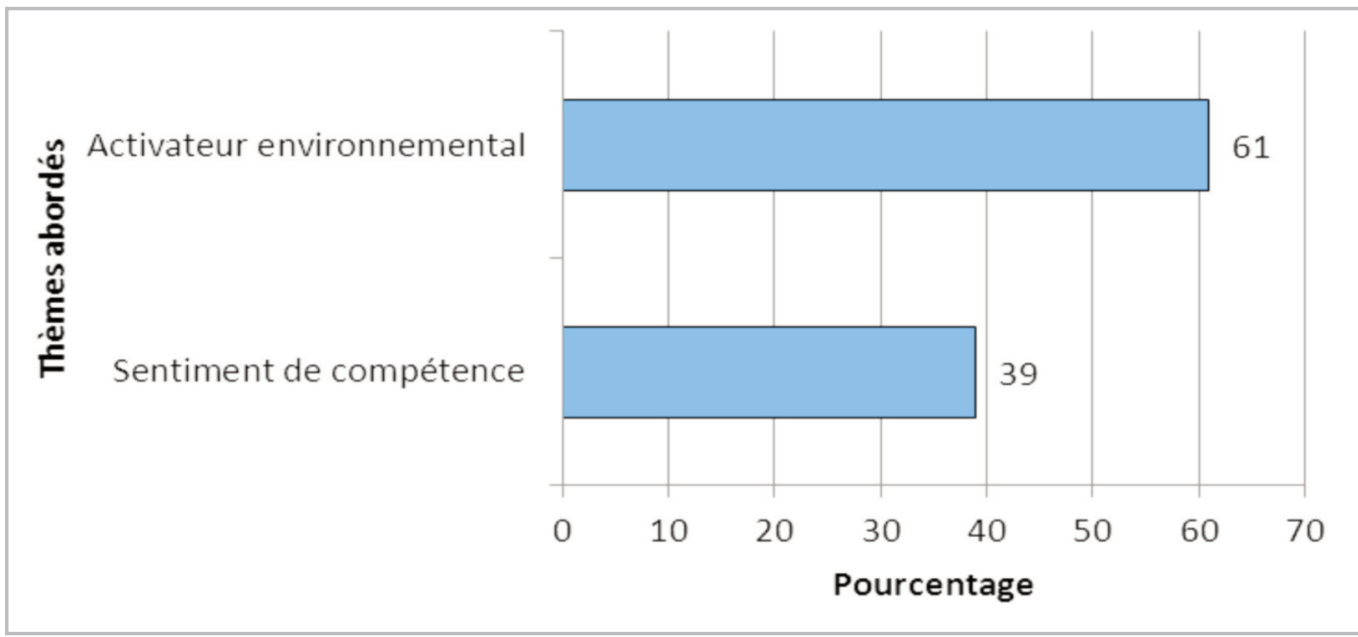

En continuité, la figure 6 montre que le sentiment de compétence (38,9\%) constitue un autre élément qui peut contribuer au développement de l'autoefficacité à intégrer les TIC. Pour les participantes, leurs sentiments au regard de leur capacité à mobiliser et à utiliser efficacement des ressources au sein d'une activité intégrant les TIC témoignent de leur sentiment de compétence et agissent sur leurs états physiques et émotionnels. L'extrait suivant témoigne du fait qu'une participante s'interroge sur son sentiment de compétence et que toutes ses questions lui font vivre du stress: «Est-ce que je vais réussir?... Est-ce que je vais être compétente là-dedans? Est-ce que je vais savoir comment aider mes élèves? [...]. C'est du stress personnel» (Lucie 245-249).

\section{Discussion et conclusion}

Les résultats sont discutés à partir de la théorie sociocognitive de l'autoefficacité et d'études antérieures. Les limites de l'étude ainsi que des pistes de recherche sont également présentées.

\section{Niveau d'autoefficacité relatif à un enseignement intégrant les TIC}

Les résultats obtenus en lien avec les attentes d'efficacité montrent que la majorité des participantes sont plutôt en accord avec l'idée qu'elles sont en mesure d'intégrer les TIC dans leur enseignement. Quant aux résultats liés aux attentes de résultats, la majorité des enseignantes sont aussi plutôt en accord avec l'idée qu'un enseignement qui intègre les TIC apporte des retombées positives. 
Ces résultats vont dans le même sens que ceux des travaux de Dussault et al. (2002), également conduits auprès d'enseignants du primaire, ainsi que ceux de Robertson et Al-Zahrani (2012), Sang et al. (2010) et Liang et Tsai (2008), menés auprès d'étudiants en enseignement. Le niveau d'autoefficacité plutôt élevé obtenu dans la présente recherche et dans les études recensées peut s'expliquer par la formation reçue. En effet, les étudiants en enseignement bénéficient, lors de leur formation initiale, d'une activité sur l'intégration pédagogique des TIC, tandis que les enseignants en exercice peuvent, comme ce fut le cas pour les participantes de la présente étude, assister à des formations continues dans le domaine des TIC. Il est donc possible que les formations reçues jouent un rôle positif sur les résultats des participants en lien avec leur intégration pédagogique des TIC.

De plus, ainsi que le suggèrent les résultats présentés dans les travaux de Liang et Tsai (2008), le fait d'avoir ou non de l'expérience en enseignement ne semble pas avoir d'influence sur l'autoefficacité relative à Internet. L'analyse des données sociodémographiques de la présente étude permet d'arriver à des résultats similaires. En effet, bien que le nombre d'années d'expérience en enseignement chez les participantes varie entre 5 et 24 ans, cela semble avoir peu d'incidence sur leur autoefficacité par rapport à un enseignement qui intègre les TIC. Par exemple, Alexandra (5 ans d'expérience) tout comme Alexia (18 ans d'expérience) sont plutôt en accord avec le fait qu'elles sont en mesure d'intégrer les TIC dans leur enseignement.

\section{Sources d'autoefficacité}

Les propos évoqués le plus souvent par les participantes concernent l'expérience active de maîtrise et les états physiques et émotionnels, suivis de ceux en lien avec l'expérience vicariante et la persuasion verbale. Les deux sources évoquées le plus souvent dans les propos des participantes (l'expérience active de maîtrise et les états physiques et émotionnels) renvoient à l'enseignante elle-même, dans son processus d'intégration pédagogique des TIC. De leur côté, les deux sources évoquées le moins souvent par les participantes (l'expérience vicariante et la persuasion verbale) font référence à d'autres acteurs.

Une piste susceptible d'expliquer ce résultat concerne le contexte de travail de l'enseignant. Même si la collaboration entre collègues est une pratique encouragée, il n'en demeure pas moins que l'essentiel de la tâche enseignante s'effectue de manière individuelle. Il est donc possible que le contexte de travail, qui prédispose l'enseignant à travailler seul, soit propice à faire davantage ressortir les sources qui font seulement appel à l'enseignant lui-même que des sources qui exigent le concours des gens qui l'entourent.

En outre, le fait que les propos des participantes se rapportent majoritairement à l'expérience active de maîtrise semble cohérent avec la théorie de Bandura (2007). En effet, ce dernier mentionne que cette source se révèle la plus influente en ce qui concerne l'autoefficacité, parce que c'est celle qui illustre le plus clairement ce que l'individu est en mesure- ou non - de rassembler, ce qui est nécessaire pour vivre des succès. 
Néanmoins, parmi les études recensées portant sur les sources qui influencent l'autoefficacité, aucune n'affiche des résultats qui concordent avec ceux obtenus (voir Al-Awidi et Alghazo, 2012; Britner et Pajares, 2006; Hodges et Murphy, 2009; Kiran et Sungur, 2012; Palmer, 2006; Usher et Pajares, 2009; Van Dinther et al., 2011; Wang et al., 2004; Zeldin et al., 2008; Zeldin et Pajares, 2000). À l'heure actuelle, il ne semble donc pas y avoir de consensus sur l'importance de chacune des sources de l'autoefficacité (Gloudemans et al., 2013). En effet, les résultats diffèrent d'une étude à l'autre, et ce, pour chacune des sources d'autoefficacité. Cette divergence relève peut-être de l'intégration de l'information. En effet, le traitement effectué par l'enseignant, concernant les informations qui proviennent des quatre sources d'autoefficacité, peut différer d'une personne à l'autre (Bandura, 2007). Ainsi, il est possible que certains enseignants accordent plus d'importance aux aspects liés à une source en particulier qu'à ceux relatifs aux autres sources.

\section{Limites et pistes de recherche}

Une limite concerne le contexte de l'étude. Cette dernière s'effectuait dans le cadre de la mise sur pied d'un journal virtuel, projet d'intégration des TIC lancé par l'équipe-école. Ce contexte a pu avoir une incidence sur les résultats obtenus puisque les enseignantes étaient, en quelque sorte, tenues d'exploiter les TIC avec leurs élèves pour contribuer au journal virtuel. Il serait souhaitable qu'une recherche similaire soit conduite dans un contexte plus naturel où les enseignants seraient libres d'enseigner en intégrant ou non les TIC, et ce, à la fréquence et avec l'envergure désirées. Les résultats seraient sans doute plus représentatifs de la réalité de terrain, telle que vécue par les enseignantes.

Le petit nombre de participantes constitue aussi une limite et rend impossible la généralisation des résultats. Comme le mentionnent Fortin, Côté et Filion (2006), la généralisation des résultats peut s'effectuer seulement lorsque l'ensemble des caractéristiques a été pris en compte. Dans ce cas, le chercheur peut spécifier de manière certaine à qui les résultats peuvent être généralisés (Fortin et al., 2006). Dans le cadre de la présente étude, le faible nombre de participantes ne permet pas de dresser un ensemble des caractéristiques exhaustif, et par conséquent, rend impossible la généralisation des résultats à d'autres milieux. En lien avec cela, il apparaît pertinent de reconduire la recherche, cette fois auprès d'un plus grand nombre de participants de diverses écoles.

D'autres pistes de recherche peuvent être considérées. Certaines études, comme celles de Morin et Dussault (1999), de Thibodeau, Dussault, Frenette et Royer (2011) ainsi que de Tollah (2003) montrent que le directeur ou la directrice d'école influencent l'autoefficacité des enseignants relativement à diverses tâches. De ce fait, il serait intéressant d'étudier l'influence du leadership de la direction d'école sur l'autoefficacité relative à un enseignement intégrant les TIC des enseignants.

En somme, l'autoefficacité représente une voie intéressante à privilégier pour accroître la compétence des enseignants à intégrer les TIC et ainsi offrir davantage d'occasions aux élèves de travailler avec les outils technologiques. Les programmes de formation initiale et continue gagneraient à développer des formules qui tiennent 
compte des sources et de leur incidence sur le développement de l'autoefficacité à intégrer pédagogiquement les TIC.

\section{Références bibliographiques}

AL-AWIDI, H. M. et ALGHAZO, I. M. (2012). The effect of student teaching experience on preservice elementary teachers' self-efficacy beliefs for technology integration in the UAE. Educational Technology Research and Development, 60(5), 923-941.

ANDERSON, S. L. et BETZ, N. E. (2001). Sources of self-efficacy expectations: Their measurement and relation to career development. Journal of Vocational Behavior, 58(1), 98-117.

BANDURA, A. (1997). Self-Efficacy: The Exercise of Control. New York, NY: Freeman.

BANDURA, A. (2007). Auto-efficacité: le sentiment d'efficacité personnelle. Bruxelles: De Boeck.

BIDJANG, S. G., GAUTHIER, C., MELLOUKI, M. et DESBIENS, J.-F. (2005). Les finissants en enseignement sont-ils compétents? Une enquête québécoise. Québec, Canada: Les Presses de l'Université Laval.

BONG, M. et SKAALVIK, E. M. (2003). Academic self-concept and self-efficacy: How different are they really? Educational Psychology Review, 15(1), 1-40.

BRITNER, S. L. et PAJARES, F. (2006). Sources of science self-efficacy beliefs of middle school students. Journal of Research in Science Teaching, 43(5), 485-499.

BURSAL, M. et YIGIT, N. (2012). Pre-service science and technology teachers' efficacy beliefs about information and communication technologies (ICT) usage and material design. Educational Sciences: Theory \& Practice, 12(2), 1084-1088.

CHAI, C. S., KOH, J. H. L., TSAI, C.-C. et TAN, L. L. W. (2011). Modeling primary school pre-service teachers' Technological Pedagogical Content Knowledge (TPCK) for meaningful learning with information and communication technology (ICT). Computers \& Education, 57(1), 1184-1193.

DUSSAULT, M., DEAUDELIN, C., BRODEUR, M. et RICHER, J. (2002). Validation de l'échelle du sentiment d'efficacité des enseignants à l'égard de l'intégration des TIC en classe (SETIC). Mesure et évaluation en éducation, 25(2-3), 1-10.

FORTIN, M.-F., CÔTÉ, J. et FILION, F. (2006). Fondements et étapes du processus de recherche. Montréal, Canada: Les Éditions de la Chenelière. 
GIBSON, S. et DEMBO, M. H. (1984). Teacher efficacy: A construct validation. Journal of Educational Psychology, 76(4), 569-582.

GLOUDEMANS, H., SCHALK, R., REYNAERT, W. et BRAEKEN, J. (2013). The development and validation of a five-factor model of sources of self-efficacy in clinical nursing education. Journal of Nursing Education and Practice, 3(3), 80-87.

GOKTAS, Y., YILDIRIM, Z. et YILDIRIM, S. (2009). Investigation of K-12 teachers' ICT competencies and the contributing factors in acquiring these competencies. The New Educational Review, 17(1), 276-294.

GRENON, V. (2007). Impact de la formation en milieu de pratique sur les stagiaires quant au développement de leur niveau d'alphabétisation informatique, de leur sentiment d'auto-efficacité et de leurs attitudes de stress et d'utilité perçue au regard des TIC. Thèse de doctorat inédite, Université de Sherbrooke.

GUZMAN, A. et NUSSBAUM, M. (2009). Teaching competencies for technology integration in the classroom. Journal of Computer Assisted Learning, 25(5), 453-469.

HAMEL, J. (2010). L'objet d'analyse comme pivot de l'analyse qualitative assistée par ordinateur. Récupéré de www.recherche-qualitative.qc.ca/revue/hors_serie/ hors_serie_v9/HS9_Hamel.pdf

HERMANS, R., TONDEUR, J., VAN BRAAK, J. et VALCKE, M. (2008). The impact of primary school teachers' educational beliefs on the classroom use of computers. Computers \& Education, 51(4), 1499-1509.

HODGES, C. B. et MURPHY, P. F. (2009). Sources of self-efficacy beliefs of students in a technology-intensive asynchronous college algebra course. Internet and Higher Education, 12(2), 93-97.

HSU, S. (2010). Developing a scale for teacher integration of information technology in grades 1-9. Journal of Computer Assisted Learning, 26(3), 175-189.

KARSENTI, T., RABY, C., VILLENEUVE, S. et GAUTHIER, C. (2007). La formation des maîtres et la manifestation de la compétence professionnelle à intégrer les technologies de l'information et des communications (TIC) aux fins de préparation et de pilotage d'activités d'enseignement-apprentissage, de gestion de l'enseignement et de développement professionnel. Montréal: CRIFPE, Université de Montréal.

KIRAN, D. et SUNGUR, S. (2012). Middle school students' science self-efficacy and its sources: Examination of gender difference. Journal of Science Education and Technology, 21(5), 619-630.

KLASSEN, R. M., TZE, V. M. C., BETTS, S. M. et GORDON, K. A. (2011). Teacher efficacy research 1998-2009. Signs of progress or unfulfilled promise? Educational Psychology Review, 23(1), 21-43. 
KREIJNS, K., VAN ACKER, F., VERMEULEN, M. et VAN BUUREN, H. (2013). What stimulates teachers to integrate ICT in their pedagogical practices? The use of digital learning materials in education. Computers in Human Behavior, 29(1), 217-225.

LAROSE, F., GRENON, V. et PALM, S. B. (2004). Enquête sur l'état des pratiques d'appropriation et de mise en ouvre des ressources informatiques par les enseignantes et les enseignants du Québec. Résumé administratif des principaux résultats de l'analyse des questionnaires et des entrevues réalisées. Sherbrooke: Centre de recherche sur l'intervention éducative. Faculté d'éducation. Récupéré de http://www.criese.ca/index.htm

LIANG, J.-C. et TSAI, C.-C. (2008). Internet self-efficacy and preferences toward constructivist Internet-based learning environments: A study of pre-school teachers in Taiwan. Educational Technology \& Society, 11(1), 226-237.

LIU, S.-H. (2011). Factors related to pedagogical beliefs of teachers and technology integration. Computers \& Education, 56(4), 1012-1022.

MELANÇON, J. (2012). Sources d'influence de l'autoefficacité relative à un enseignement intégrant les TIC chez des enseignants du primaire. Mémoire de maîtrise inédit. Trois-Rivières, Canada: Université du Québec à Trois-Rivières.

MINISTÈRE DE L'ÉDUCATION DU QUÉBEC - MEQ (2001). La formation à l'enseignement. Les orientations, les compétences professionnelles. Québec: MEQ.

MINISTÈRE DE L'ÉDUCATION, DU LOISIR ET DU SPORT - MELS (2010). Les technologies de l'information et de la communication (TIC) pour la formation générale des jeunes. Édition 2009 de l'enquête. Québec: MELS.

MORIN, R. et DUSSAULT, M. (1999). Leadership de la direction et sentiment d'autoefficacité des enseignants. Cahiers de la recherche en éducation, 6(3), 373-392.

MUELLER, J., WOOD, E., WILLOUGHBY, T., ROSS, C. et SPECHT, J. (2008). Identifying discriminating variables between teachers who fully integrate computers and teachers with limited integration. Computers \& Education, 51(4), 1523-1537.

PALMER, D. H. (2006). Sources of self-efficacy in a science methods course for primary teacher education students. Research in Science Education, 36(4), 337-353.

PAMUK, S. et PEKER, D. (2009). Turkish pre-service science and mathematics teachers' computer related self-efficacies, attitudes, and the relationship between these variables. Computers \& Education, 53(2), 454-461.

PARASKEVA, F., BOUTA, H. et PAPAGIANNI, A. (2008). Individual characteristics and computer self-efficacy in secondary education teachers to integrate technology in educational practice. Computers \& Education, 50(3), 1084-1091. 
RABY, C. (2004). Analyse du cheminement qui a mené des enseignants du primaire à développer une utilisation exemplaire des technologies de l'information et de la communication (TIC) en classe. Thèse de doctorat inédite, Université du Québec à Montréal.

ROBERTSON, M. et AL-ZAHRANI, A. (2012). Self-efficacy and ICT integration into initial teacher education in Saudi Arabia: Matching policy with practice. Australasian Journal of Educational Technology, 28(7), 1136-1151.

SANG, G., VALCKE, M., VAN BRAAK, J. et TONDEUR, J. (2010). Student teachers' thinking processes and ICT integration: Predictors of prospective teaching behaviors with educational technology. Computers \& Education, 54(1), 103112.

SHIUE, Y.-M. (2007). Investigating the sources of teachers' instructional technology use through the decomposed theory of planned behavior. Journal of Educational Computing Research, 36(4), 425-453.

THIBODEAU, S., DUSSAULT, M., FRENETTE, É. et ROYER, N. (2011). Solitude professionnelle d'enseignants du secondaire: relations avec le leadership du directeur d'école et leurs croyances d'efficacité sociale. Revue canadienne de l'éducation, 34(4), 177-199.

TOLLAH, H. (2003). Le leadership transformatif de la direction d'école, le sentiment d'autoefficacité et la motivation du personnel enseignant. Thèse de doctorat inédite, Université du Québec à Trois-Rivières et Université du Québec à Montréal, Canada.

TSAI, C. C. et CHAI, C. S. (2012). The "third"-order barrier for technologyintegration instruction: Implications for teacher education. Australasian Journal of Educational Technology, 28(Special issue, 6), 1057-1060.

USHER, E. L. et PAJARES, F. (2006). Sources of academic and self-regulatory efficacy beliefs of entering middle school students. Contemporary Educational Psychology, 31(2), 125-141.

USHER, E. L. et PAJARES, F. (2009). Sources of self-efficacy in mathematics: A validation study. Contemporary Educational Psychology, 34(1), 89-101.

VAN ACKER, F., VAN BUUREN, H., KREIJNS, K. et VERMEULEN, M. (2011). Why teachers use digital learning materials: The role of self-efficacy, subjective norm and attitude. Education and Information Technologies. Récupéré de link.springer.com.biblioproxy.uqtr.ca/article/10.1007/s10639-011-91819/fulltext.html

VAN DER MAREN, J.-M. (1996). Méthodes de recherche pour l'éducation (2 éd.). Montréal: Les Presses de l'Université de Montréal.

VAN DINTHER, M., DOCHY, F. et SEGERS, M. (2011). Factors affecting students' self-efficacy in higher education. Educational Research Review, 6(2), 95-108. 
VILLENEUVE, S., KARSENTI, T., RABY, C. et MEUNIER, H. (2012). Les futurs enseignants du Québec sont-ils technocompétents? Une analyse de la compétence professionnelle à intégrer les TIC. Revue internationale des technologies en pédagogie universitaire / International Journal of Technologies in Higher Education, 9(1-2), 78-99.

WANG, L., ERTMER, P. A. et NEWBY, T. J. (2004). Increasing preservice teachers' selfefficacy beliefs for technology integration. Journal of Research on Technology in Education, 36(3), 231-250.

WHEATLEY, K. F. (2005). The case for reconceptualizing teacher efficacy research. Teaching and Teacher Education, 21(7), 747-766.

ZELDIN, A. L., BRITNER, S. L. et PAJARES, F. (2008). A comparative study of the selfefficacy beliefs of successful men and women in mathematics, science, and technology careers. Journal of Research in Science Teaching, 45(9), 1036-1058.

ZELDIN, A. L. et PAJARES, F. (2000). Against the odds: Self-efficacy beliefs of women in mathematical, scientific, and technological careers. American Educational Research Journal, 37(1), 215-246. 